\title{
Computational Fluid Dynamic Analysis of the Flow though T-junction and Venturi Meter
}

\author{
Enas Salman Taha', Mohammed A. Abdulwahid ${ }^{2}$, Akeel M. Ali Morad ${ }^{3}$ \\ , Qusay A Maatooq ${ }^{4}$ \\ \{umabody.1979@gmail.com ${ }^{1}$, mohw2016@stu.edu.iq ${ }^{2}$, akeel@stu.edu.iq ${ }^{3}$ \}
}

\author{
Southern Technical University, Basra Eng. Technical College, Thermal \\ Mechanical Engineering and Fuel Energy Deartmentt ${ }^{1,2,3,4}$
}

\begin{abstract}
This study investigates the influence of turbulent parameters on the characteristic centreline of a fluid flow through a T-junction connected to a Vinturi tube through a pipe. Continuity equation, Momentum equation, and Energy equation of water are modelled and solved by using the ANSYS FLUENT 2020R1 Software free Demo. While the turbulent model with standard (k-e) type is used to compute the turbulent parameters such as turbulent kinetic energy and turbulent dissipation rate for a pipe inner diameter of $(\mathrm{D}=25 \mathrm{~mm})$ with a length from the $\mathrm{T}$-junction centre line and at the outlet of the Venturi meter is $(350 \mathrm{~mm})$. The Reynolds number variation ranged from $\left(1 \times 10^{4}\right.$ to $\left.3 \times 10^{4}\right)$ at the step of (5000). The water flowing is considered in an $\mathrm{x}$-direction flow to show the effects of the above parameters. The model is obtained on the velocity distribution, pressure drop distribution, turbulent kinetic energy, and turbulent dissipation rate. The results showed a discrepancy in the values of the effect of the T-junction on theVenturi meter concerning the velocity distribution and pressure drop, while they showed similar behaviour for the turbulence parameters.
\end{abstract}

Keywords: CFD, Turbulent Flow, Characteristics centreline, T-junction, Venturi meter.

\section{Introduction}

When flowing in pipes and ducts, it encounters resistance to its flow. In pipes, this resistanceis represented by the roughness of the pipe surface and friction losses, and there are other losses due to the presence of gate valves, ball valves, sudden expansions, or connections such as $\mathrm{T}$-junction, and all these factors affect the values of velocity, pressures, and other parameters of the flow.

There are two basic types of flow in pipes, depending on the nature of the different components outlined above that affect the flow. The first form is laminar flow, which is defined by fluid passing across smooth layers of laminae. This form of fluid motion is known as streamline flow because all of the fluid particles travel in parallel lines.

When the flow velocity reaches a particular point, the flow becomes turbulent rather than laminar. A particle's mobility becomes random in a turbulent flow and fluctuates up and down in a perpendicular and parallel to the mean flow direction. The Reynolds numbers $(\mathrm{Re})$ are important in assessing whether the flow pattern in a pipe is laminar or turbulent [1]. Pipe networks depend on T-junction, a common component of water and industrial systems. Divide (diverge) the flow from the main pipe to multiple branch streams and gather (converge) flows from two pipes. The energy losses produced by the combining and division of flow at tees 
should be considered when planning and analysing piping systems. Line sizing necessitates a precise estimate of energy losses at junctions, which can have a major impact on hydraulic calculations and, in some situations, pump selection [2]. The Venturi will need to be calibrated in a laboratory using the identical installation setup as it will be in the field to reliably measure flow. Depending on the Venturi size and piping layout, this calibration may or may not be possible [3]. Around 1791, Giovanni B. Venturi discovered the Venturi tube. When a Venturi tube is used for flow metering, a pressure differential is created dependent on the rate of flow, which is known as a Venturi metre. Venturi meters measure the flow rate by measuring the increase in flow velocity and corresponding pressure drop due to the converging section of the pipe. Water supply companies have been using them for many years. Analysed the effect of a tee junction on the measurement of the flow rate of a Venturi flow meter constructed downstream of the branch. Results suggest that the measuring of flow rate was less influenced when all flow wasentering the tee branch and more affected when less flow entered to its. The difference between experimental and numerical results increases as the flow split entering the Venturi decreases [4]. It is dependent on the Venturi installation experimental and analytical study about the pressure loss of a fluid with turbulent incompressible flow through a $90^{\circ}$ tee junction. The study found at various Reynolds numbers, the effect of the flow rate ratio q (ratio between the flow rate in the branch and outlet pipes) on pressure drop and velocity profile was expected. It is also the pressure and total energy losses arise as the flow rate ratio is increased. A study by [5] was conducted to measurements of pressure variation, mean, and turbulent velocities were forthe flow of water in two $90^{\circ}$ tee junctions with sharp and rounded edges. It was found the losscoefficient of the branched flow was greater than that of the straight flow. A study conducted by [6] resulted in the flow rate ratio has a significant effect on the pressure loss and then the outlet flow rate, also it was found the pressure loss coefficient $\mathrm{km}$ depends upon the flow rateratio q, but not upon the Reynolds. [7] A steady, incompressible fluid flow was examined with CFD through a T-junction. Water velocity is strongly affected by the 90 -degree angles of the T-junction of the pipe, water velocity increases significantly with decreasing diameter of the test section, and energy loss can be reduced by reducing the angle of the Tjunction of thepipe. In a study by [8], computational parametric analysis of turbulent flow in 90 T-junctions with sharp and curved corners was performed. In the main pipe (K32), the loss coefficient is the same in both instances, but it is considerably lower in the branch pipe (K31). To determine the effect of pipe size and beta values on discharge coefficients, various models of the flow meters were studied. For typical Reynolds numbers, the Venturi, V-cone, and wedge flow meters all have relatively constant discharge coefficients. Studying the influences of geometricparameters such as convergent cone angle, divergent cone angle, beta ratio, and throat length on pressure drop, [9] found that beta ratio was the most significant parameter. [10] Have studied CFD and laboratory data to determine the relationship between the classical Venturi meter design's recovery cone angle and associated head loss. As a result, the beta ratio and Reynolds number determine the best recovery cone angle for minimising head loss. The purpose of [11] was to assess the performance of Venturi Meters positioned downstream of the bend. They discover that the length of straight pipe required is determined by the meter's upstream fitting type and the ratio of the throat to inlet diameter. The accuracy of a Venturi metre is dependent on the length of straight pipe upstream of the metre.

The above-mentioned kinds of literature have done studies on the Tee Junction or the Venturi meter separately. But in this study, the objective was to determine the effects of different turbulent Reynolds numbers entering the T-junction on the performance of the Venturi meter based on the method of CFD. 


\section{Description of the Venturi Meter}

A typical Venturi tube made from a transparent material (Epoxy) calibrated in water with diameter ratio $\beta=0.6$ was derived from [12]; the convergent and the divergent angles were set at 10.5 and 3.5, respectively. The length of the inlet cylinder is equal to the internal diameter of the branch pipe $D=25 \mathrm{~mm}$, the length of the throat section is equal to the internal diameterof throat $d=15 \mathrm{~mm}$, the divergent section length is $82 \mathrm{~mm}$. Venturi meter length is $150 \mathrm{~mm}$, upstream pipe length is $8 \mathrm{D}$.

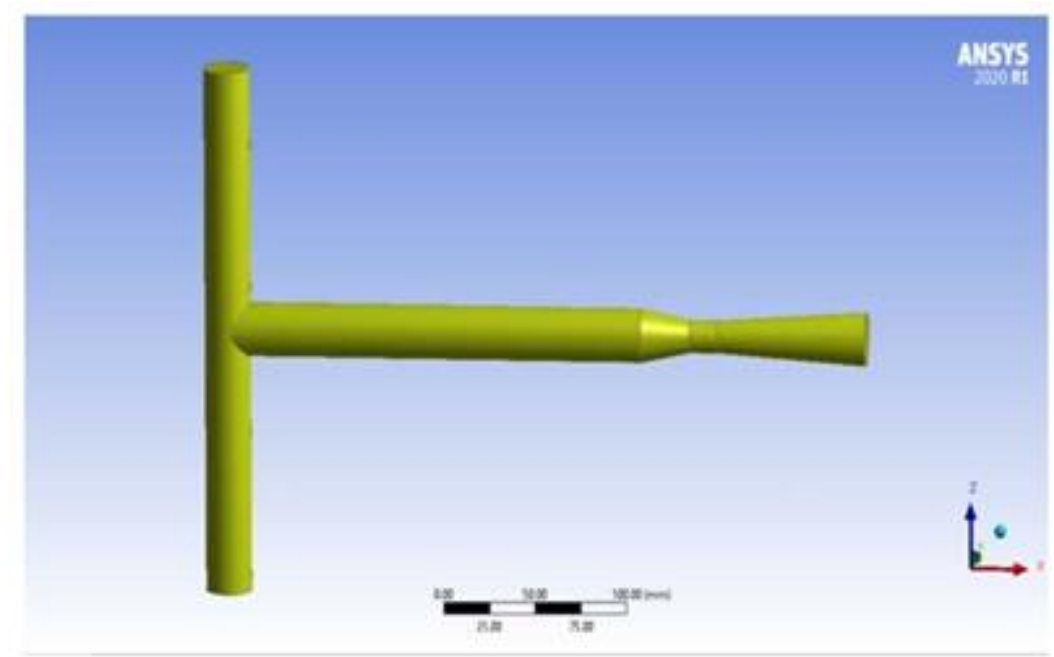

Fig 1. The geometry of the T-junction \& Venturi meter.

\section{Mathematical Model}

The water flowing through the T-junction and Venturi metre can be thought of as an incompressible, steady flow, which is in accordance with mass and momentum conservation laws. Therefore, the continuity and the Navier-Stokes equations are included in the basic equations [13]:

$$
\frac{6 u}{6 x}+\frac{6 v}{6 y}+\frac{6 w}{6 z}=0
$$

Where $\mathrm{u}, \mathrm{v}, \omega$ is the velocity components in $\mathrm{x}, \mathrm{y}, \mathrm{z}$-direction, respectively.

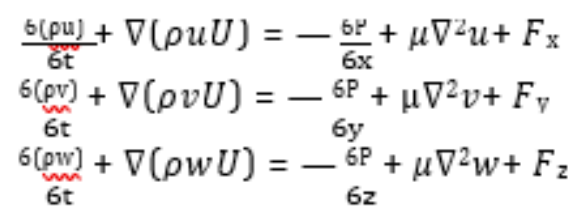

Where $t$ is time, $U$ is velocity vector, $\rho$ is the water density, $p$ is the pressure of the fluid, $\mu$ is the dynamic viscosity of water, Fx, Fy, Fz respectively. When the mass force is only the gravity and Zaxis is vertical, then comes $\mathrm{Fx}=0, \mathrm{Fy}=0$, and $\mathrm{Fz}=-\rho \mathrm{g}$.

Both turbulent kinetic energy and turbulent dissipation rate can calculate [14]. 


$$
\left.k=\left(\mu+\mu_{\mathrm{T}}\right)\left(V_{\mathrm{u}}+\left(\mathrm{V}_{\mathrm{u}}\right)^{1}\right)-{ }^{2} \frac{1}{3} \mu+\mu_{\mathrm{T}}\right)\left(V_{\mathrm{u}}\right)-{ }^{2}{ }_{3} K_{\mathrm{T}}
$$

Where $\mathrm{K}$ is turbulent kinetic energy, $\epsilon$ is turbulent dissipation rate.

Reynolds number ranged (from $1 \times 10^{4}$ to $3 \times 10^{4}$ ) and the flow section changed. For numerical simulation, the standard $(\mathrm{k}-\varepsilon)$ type of turbulent model is used. In the $(\mathrm{k}-\varepsilon)$ turbulence model, turbulent kinetic energy is $\mathrm{k}$, dissipation ratio is $\varepsilon$.

\section{Flow Parameters}

\subsection{Flow Geometry}

The T-junction and Venturi meter are drawn using the Cartesian coordinate system presentedin Fig 1. The diameter of the pipe used $(\mathrm{D}=0.025 \mathrm{~m})$, the origin of the coordinate system is located at the beginning of the throat section therefore the length of the throat section is from $(0-0.015 \mathrm{~m})$, the convergent section is from $\left(0_{-}-0.025 \mathrm{~m}\right)$, the entrance section from $(-0.025--0.05 \mathrm{~m})$. The upstream of Venturi meter is $8 \bar{D}$ from $(-0.05 \ldots-0.25 \mathrm{~m})$ on characteristic centreline of $\mathrm{X}$-axis as in Fig 3.

\subsection{Simulation Paramters}

The fluid used in the simulation is water with a constant density of $998 \mathrm{~kg} / \mathrm{m}^{3}$ and $0.001 \mathrm{~kg} / \mathrm{ms}$ dynamic viscosity. The fluid flow is assumed incompressible. The boundary conditionsare set as a velocity at the inlet and zero initial gauge pressure. The velocity of the inlet pipe isfully developed. Assumed that all the walls have no-slip boundary conditions. The simulation analysis is carried out using the CFD software ANSYS FLUENT2020 R1. Using the finite volume method and a first-order upwind difference scheme in this software, differential equations might be reduced to algebraic equations. The discrete equations' pressure-velocity rate is calculated using the coupling procedure. The unstructured computational grid (tetrahedron) shown in Figure (2) is made up of 767487 nodes and 2431372 elements, with five boundary layer cells and an element size of $0.75 \mathrm{~mm}$.

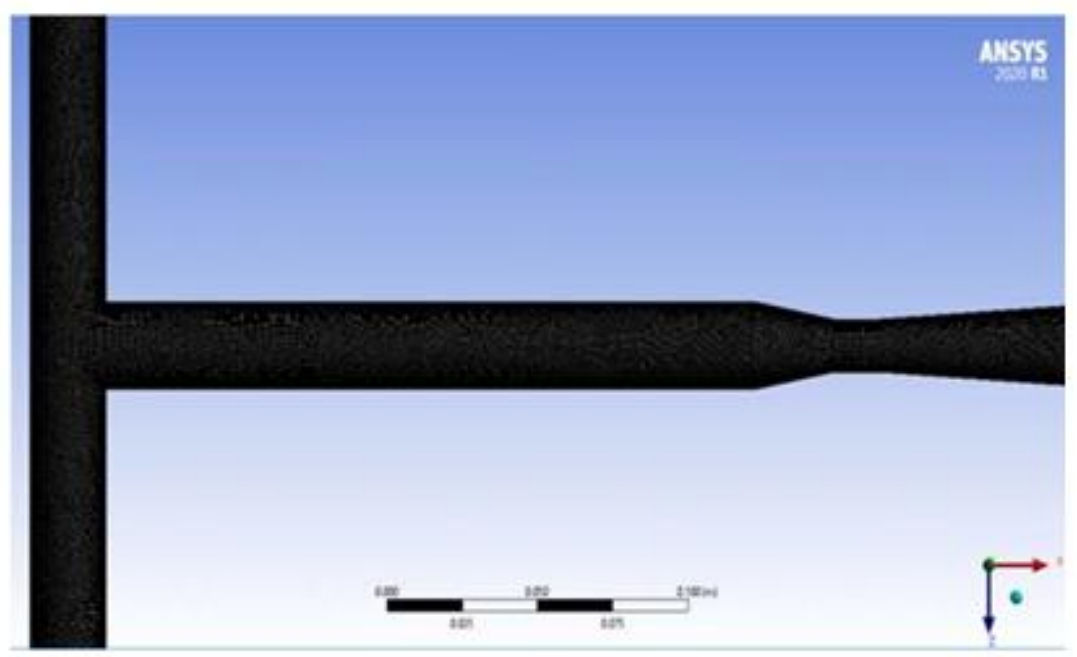

Fig 2. The unstructured mesh for T-junction \&Venturi meter. 


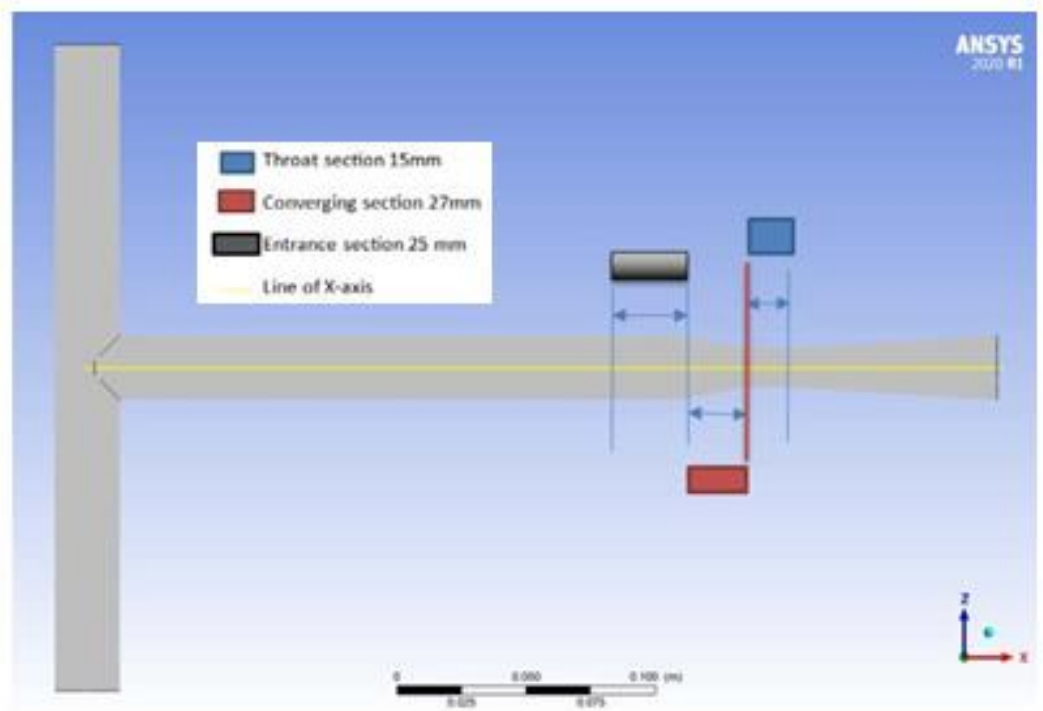

Fig 3. The geometry plotted of characteristics centreline.

\section{Results and Discussion}

A characteristic line is plotted from the centre of the T-junction extended to the end of the Venturi meter to describe the effects of the T-junction on the water flow through the Venturi. The length of this line is $(350 \mathrm{~mm})$ as shown in Figure 3. The CFD analysis for turbulent flow with the $(\mathrm{k}-\varepsilon)$ standard method is used to show the effects of the velocity distribution, pressure distribution, turbulent kinetic energy and dissipation rate for Reynolds numbers of ranges (from $1 \times 10^{4}$ to $3 \times 10^{4}$ ) with an average of 5,000 steps. The velocity distribution is shown in Fig 4 when flow enters the pipe after the $\mathrm{T}$ - junction, the velocity suddenly drops and remains almost constant along the length of the pipeuntil entering the Venturi section. We notice a gradual change in velocity, especially in the throat section, due to the reduction in the area, so the velocity increases.

Fig 5 illustrates the behaviour of the pressure distribution of the characteristic line as a result of friction through the $\mathrm{T}$-junction and beyond. As the Reynolds number increases, the pressure drop in the connected pipe increases until it reaches its lowest value ( $-45 \mathrm{pa})$ at the point (1.5 $\mathrm{D})$ of the pipe, then the drop decreases until it reaches $(15 \mathrm{~Pa})$ at the point $(8 \mathrm{D})$ at the entrance to the venturi tube, at the throat, it reaches $(0 \mathrm{~Pa})$ and then begins to drop to reach $(-15 \mathrm{~Pa})$ at the end of the throat section for $(\operatorname{Re}=30000)$. 


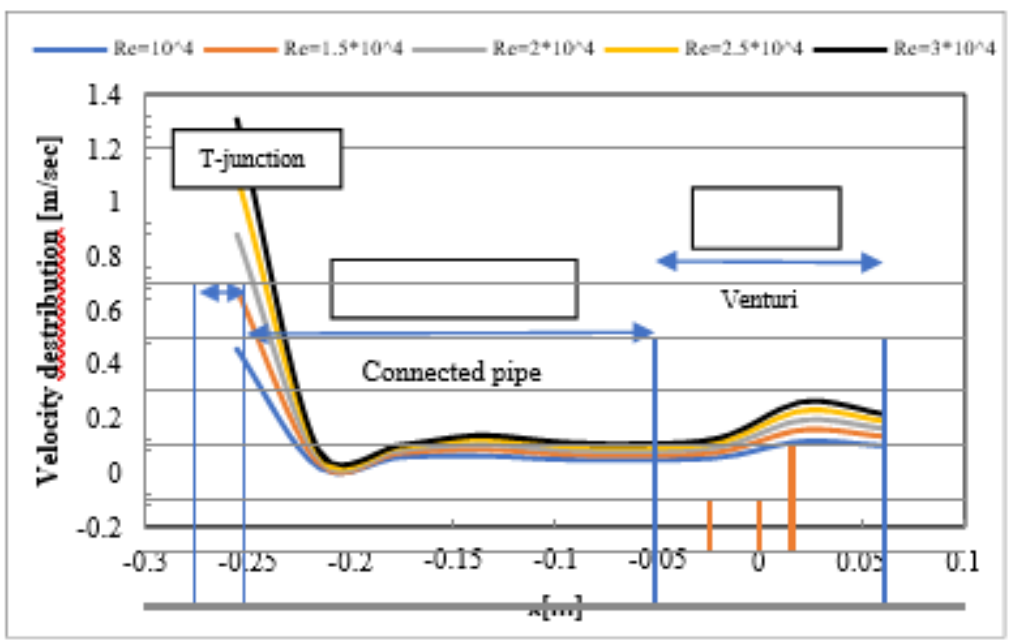

Fig 4. Velocity distribution of the characteristic line at five Reynolds numbers.

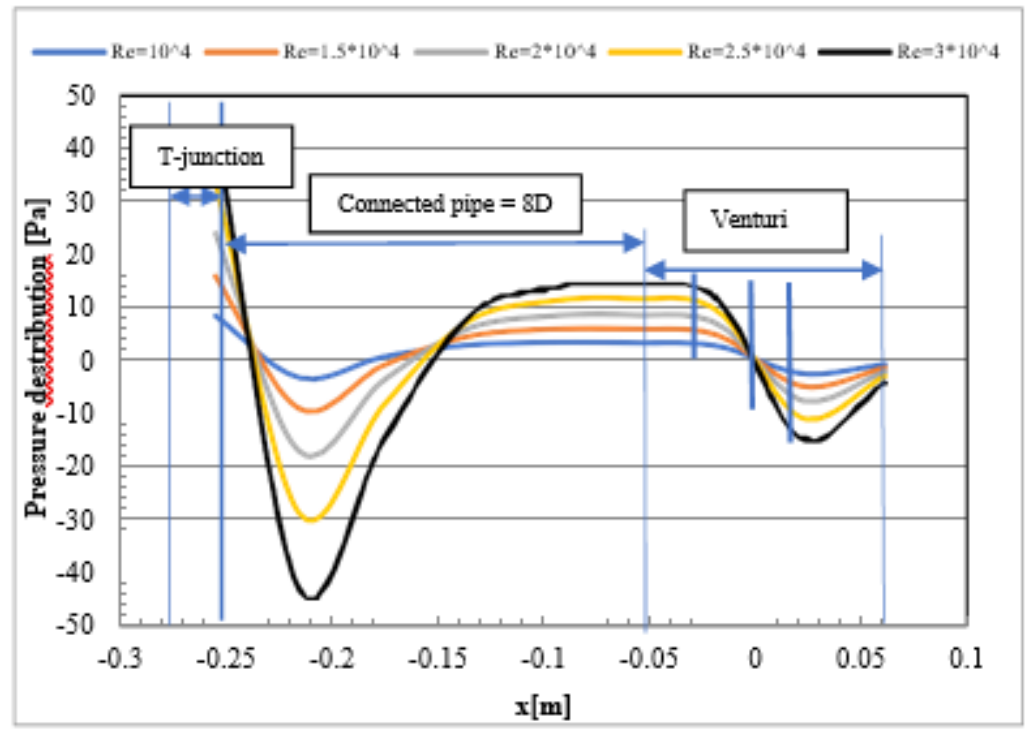

Fig 5. Pressure drops distribution of the characteristic line at five Reynolds numbers.

Under turbulent flow conditions inside the conduct's channels such as pipes, fittings, and so on. There are two important parameters notified to predict the power reflected in the fluid flow.

Turbulent kinetic energy describes the amount and behaviour of the transformed energy to the fluid inside the conducted channels, while the turbulent dissipation rate is given the amount of energy deformed into the flow stream.

Figs 6 and 7 explained the turbulent kinetic energy and the dissipation rate respectively. Same behaviour of the two parameters along the characteristic line of the flow, thetwo figures show a very slight chance of the turbulence parameters through the Venture tube, while a very clear change in the values appears along the connected pipe, as the higher the Reynolds number, increase the effect on the values through the connected pipe. 


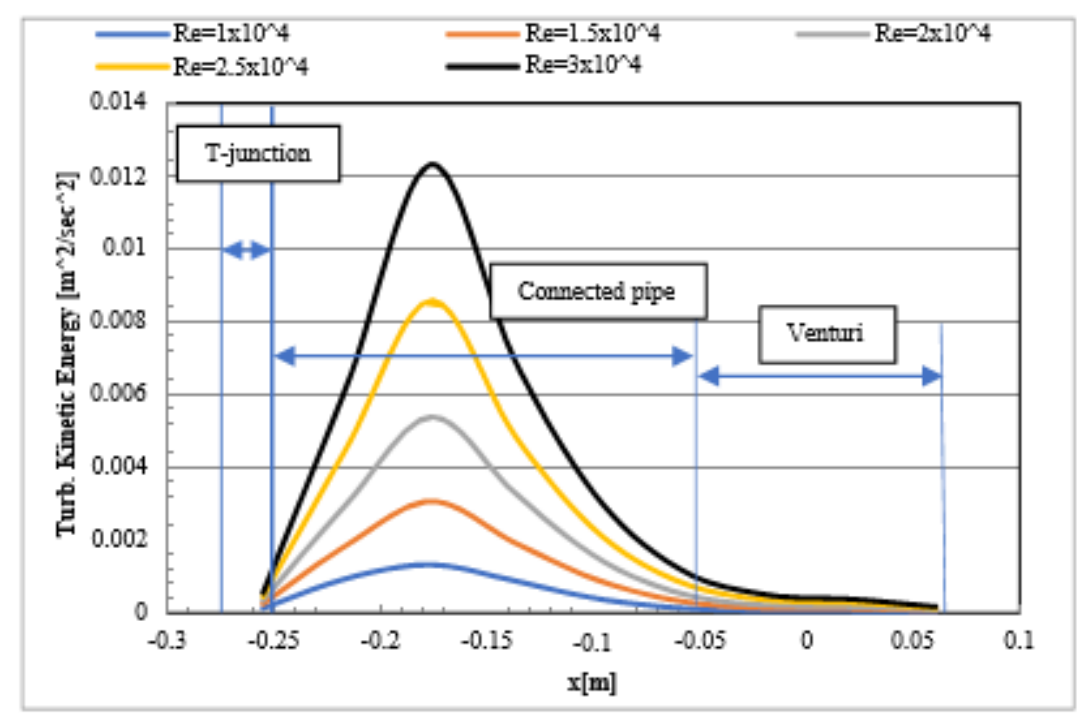

Fig 6. The turbulent kinetic energy of the characteristic line at five Reynolds numbers.

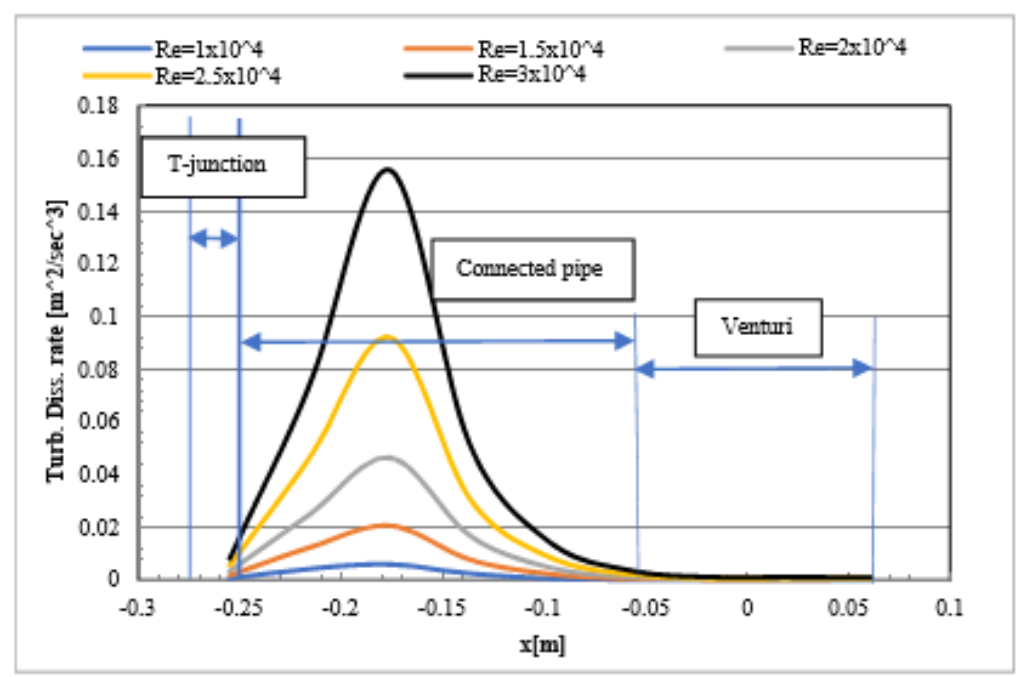

Fig 7. Turbulent dissipation rate of the characteristic line at five Reynolds Number.

\section{Conclusion}

Five turbulent Reynolds numbers are used to investigate the behaviour of the water flowing from the centreline of the T-junction to the outlet of the Venturi. This line is called the characteristic centreline of water flowing, through this line all changes of parameters related to turbulent flow can be discovered. It was concluded by changing the Reynolds number for various values which lead to show the behaviour of each of the velocity distribution, pressure drop distribution, turbulent flow parameters, turbulent kinetic energy, and dissipationrate. So, the conclusions can be explained as follows:

1. For the velocity distribution, the velocity is nearly uniform along the connecting pipe, 
but it suffers from a significant decrease at the T-junction and a relative change through the Venturi tube due to the change in the cross-sectional area through it.

2. As for the pressure distribution, there is a significant fluctuation in the pressure drop along the characteristic line, which becomes more apparent as the Reynolds number increases.

3. Turbulent kinetic energy and dissipation rate are two important characteristics of turbulent flow. These variables changed the turbulent conditions, allowing researchers to find out how much the water's flow power changed. The results show the same behaviour for an increase in the Reynolds number, which is associated with the changes in these parameters.

\section{References}

[1] R. Gürbüz, "Measuring local losses in pipe flows utilising data acquisition cards and acomputer," vol. 2, no. 3, pp. 461-464, 2003.

[2] C. T. Michalos, "A Review of Available Minor Loss Coefficients for Combining and Dividing Flow Tees," pp. 1116-1123, 2011.

[3] Benjamin G. Sandberg, "VENTURI FLOWMETER PERFORMANCE INSTALLED DOWNSTREAM OF THE BRANCH OF A TEE JUNCTION," 2020.

[4] S. F. D. Mohammed Abdulwahhab, Niranjan Kumar Injeti, "Numerical prediction of pressureloss of fluid in a T-junction," Int. J. Energy Environ., vol. 4, no. 2, pp. 253-264, 2013.

[5] M. F. P. N. P. Costa, R. Maia, F. T. Pinho, "EDGE EFFECTS ON THE FLOW CHARACTERISTICS IN A 90 TEE JUNCTION," pp. 1-44, 2006.

[6] G. B. Nimadge and S. V Chopade, "CFD ANALYSIS OF FLOW-THROUGH T-JUNCTIONOF PIPE," no. 2, 2017.

[7] G. Paál, F. Pinho, and R. Maia, "THE EFFECT OF CORNER RADIUS ON THE ENERGYLOSS IN $90^{\circ} \mathrm{T}$ - JUNCTION TURBULENT FLOWS,” 2006.

[8] C. L. Hollingshead, M. C. Johnson, S. L. Barfuss, and R. E. Spall, "Discharge coefficient performance of Venturi, standard concentric orifice plate, V-cone and wedge flow meters at low Reynolds numbers," J. Pet. Sci. Eng., vol. 78, no. 3-4, pp. 559-566, 2011, DOI: 10.1016/j.petrol.2011.08.008.

[9] C. Sanghani and D. Jayani, "Optimization of Venturimeter Geometry for Minimum PressureDrop using CFD Analysis," vol. 3, no. 2, pp. 31-35, 2016.

[10]Z. B. Sharp, M. Asce, M. C. Johnson, M. Asce, S. L. Barfuss, and M. Asce, "Optimizing the ASME Venturi Recovery Cone Angle to Minimize Head Loss," vol. 144, no. 1, pp. 1-9, 2018, DOI: 10.1061/(ASCE)HY.1943-7900.0001387.

[11]M. C. Johnson and J. G. Gilbert, "Performance of Venturi Meters," no. April, pp. 156-164, 2006, DOI: 10.1002/j.1551-8833.2006.tb07643.x.

[12]R. L. Wakeland, “A study of venturi tubes,” Int. Conf. Nucl. Eng. Proceedings, ICONE, vol. 4,no. 1, pp. 885-892, 2012, DOI: 10.1115/ICONE20-POWER2012-55212.

[13]H. Reza and M. Reza, "Optimizing Different Angles of Venturi in Biodiesel Production UsingCFD Analysis," vol. 38, no. 6, pp. 285-295, 2019.

[14]Akeel M. Ali Morad, Rafi M. Qasim, Amjed Ahmed Ali (2020). STUDY OF THE BEHAVIOURS OF SINGLE-PHASE TURBULENT FLOW AT LOW TO MODERATE REYNOLDS NUMBERS THROUGH A VERTICAL PIPE. PART I: 2D COUNTERS ANALYSIS. (2020), «EUREKA: Physics and Engineering» Number 6. PP.108-122. DOI: $10.21303 / 2461-4262.2020 .001538$. 